\title{
MIEDO, ANSIEDAD Y AFRONTAMIENTO ANTE LA MUERTE EN ESTUDIANTES DE ENFERMERÍA: EFECTOS DE UN PROGRAMA DE FORMACIÓN
}

\author{
FEAR, ANXIETY AND COPING WITH THE DEATH OF NURSING \\ STUDENTS: EFFECTS OF TRAINING PROGRAM
}

Recibido: 27/04/2015

Revisado: 12/05/2015

Aceptado: 05/06/2015

Marta Linares Manrique ${ }^{1}$

\section{RESUMEN}

Objetivo: Evaluar en una población de estudiantes de enfermería de la península del Yucatán, la ansiedad, el afrontamiento y el miedo ante la muerte en relación con diversas variables sociodemográficas: sexo, curso académico, y formación o ausencia de ella en tanatología, así como la influencia de un programa de intervención. Material y métodos: El estudio que se presenta es de carácter cuantitativo y causi-experimental. La muestra quedo constituida por la totalidad de sujetos que pertenecen a la población de referencia $\mathrm{N}=274$. Resultados: De manera general, tras la implementación de nuestro programa específico acerca de la muerte y su proceso, los alumnos del grupo experimental han modificado el miedo, la ansiedad y el afrontamiento de manera positiva, por otra parte y tal y como se esperaba, aquellos integrantes pertenecientes al grupo control, no han modificado sus parámetros iniciales en cuanto a las actitudes referidas. Conclusiones: Luego el tratamiento de datos, podemos mencionar que nuestro programa específico acerca de la muerte y su proceso, es efectivo para cambiar las actitudes que se presentan cuando la persona se tiene que enfrentar a la muerte, en nuestra población de estudio.

Palabras clave: Muerte, enfermería, cuidados paliativos.

\section{ABSTRACT}

Objective: To evaluate in a population of nursing students from the Yucatan Peninsula, anxiety, coping and the fear of death in relation to sociodemographic variables: sex, academic year and training or lack thereof in tanatología and the influence of an intervention program. Material and methods: The study presented is quantitative and Causiexperimental. The sample was composed of all subjects belonging to the reference population $N=274$. Results: In general, after the implementation of our specific program about death and its process, the experimental group students have modified the fear, anxiety and coping positively, on the other hand and as expected, those belonging to the control group members, has not changed its initial parameters regarding the aforementioned attitudes. Conclusions: After data processing, we mention that our specific program about death and its process, it is effective to change the attitudes that arise when a person has to face death in our study population.

Keywords: Death, nursing, palliative care.

\section{INTRODUCCIÓN}

En la sociedad actual y debido a los adelantos tecnológicos y médicos, las formas de morir han cambiado (Sanz-Ortiz, 2006), de hecho se ha puesto de manifiesto, el que los gobiernos y los sistemas de salud, deban de establecer nuevos modelos sanitarios que se adapten a las necesidades y demandas que la sociedad pide y legislar de acuerdo con ellas (Gálvez, 2011).

La institucionalización hospitalaria o el cambio producido en el manejo de la comunicación entre médico, paciente y familia, han propiciado el cambio en las actitudes hacia la muerte, lo que desemboca en que puedan aparecer en los individuos respuestas negativas como la ansiedad, el miedo, el rechazo o la falta de afrontamiento ante la muerte, lo que ocasiona que la muerte se convierta en uno de los grandes tabús del siglo XXI.( Gala-León et al., 2002; GarcíaCaro, 2008).

Según Lamberth (1982), la actitud se puede definir como una respuesta evaluativa, relativamente estable, en relación con un objeto, que tiene consecuencias cognitivas, afectivas y probablemente comportamentales. En un lenguaje más cotidiano, el concepto de actitud se puede atribuir a una postura que expresa una intención o emoción, de forma más general, una visión del mundo (Siracusa 2010). De lo referido, se puede mencionar que las actitudes que tenemos ante cualquier hecho de la vida diaria, incluida la muerte y el morir, dan muestra de lo que pensamos, sentimos y posteriormente, mediante una conducta reflejamos a la sociedad. Por tanto, un sujeto puede sentir algo como positivo y no actuar de acuerdo con este sentimiento. (Neimeyer, 1997).

En este sentido cabe mencionar la importancia que la formación específica puede tener en los estudiantes de enfermería, ya que existen estudios en los que se manifiesta que una de las experiencias más estresantes para los alumnos, durante las prácticas clínicas, es el enfrentarse a la muerte (Benbunan et al., 2007). En la literatura consultada se manifiesta que una formación específica en cuidados al final de la vida, modula la respuesta emocional de aquellos sujetos que han participado en programas de formación ante la muerte y el morir, provocando que se enfrente el personal sanitario, a la práctica clínica de manera más efectiva, ya que estos, mejoran su afrontamiento ante la muerte, a la par que se sienten mejor cuando trabajan con pacientes en situación de terminalidad (Mason y Ellershw, 2010).

La investigación tiene como objetivo general, evaluar en una población de estudiantes de enfermería de la península del Yucatán, la ansiedad, el afrontamiento y el miedo ante la muerte en relación con diversas variables 
sociodemográficas: sexo, curso académico, y formación o ausencia de ella en tanatología, así como la influencia de un programa de intervención.

Dentro de sus objetivos específicos se resaltan: adaptar las escalas y comprobar su fiabilidad en la población de estudiantes de enfermería de la península del Yucatán; comprobar los efectos de un programa de intervención en la ansiedad ante la muerte y el proceso de morir de la población objeto de estudio, comprobar los efectos de un programa de intervención en el miedo ante la muerte y el proceso de morir de la población objeto de estudio; y finalmente, comprobar los efectos de un programa de intervención en el afrontamiento ante la muerte y el proceso de morir de los estudiantes de enfermería de la península del Yucatán.

\section{MATERIALES Y MÉTODOS}

El estudio que se presenta es de carácter cuantitativo y causi-experimental.

La población de referencia para la realización de la investigación fue la de la totalidad de sujetos de la Licenciatura de Enfermería que cursaban el primer semestre del curso académico 2012-2013.

La muestra quedó constituida por la totalidad de sujetos que pertenecen a la población de referencia $N=274$, y que cumplían como criterios de inclusión, el haber firmado el consentimiento informado en el que se les informaba del objeto del estudio, los mecanismos de confidencialidad y la aceptación de las condiciones del mismo, y que su edad cumplida fuese superior a $17 \mathrm{e}$ inferior a 25 años.

Previa a la realización del estudio se solicitó y fue concedido el permiso pertinente al comité ético de la escuela universitaria.

La recogida de datos se realizó en el mes de diciembre del curso académico 2012-2013. Los participantes contestaron a un cuestionario autoadministrado en el que se incluía:

Un cuestionario que recogía datos de carácter sociodemográficos: sexo, edad, semestre o ciclo que cursan, formación en tanatología, estado en que residen y el estado de nacimiento de los sujetos así como el de sus padres y abuelos.

$Y$ tres cuestionarios previamente validados $y$ utilizados en lengua inglesa y en español. Que se validaron previos a la implementación del programa formativo para la población del estudio.

- La Escala Revisada de Ansiedad ante la Muerte de Thorson y Powell (1992).

- Escala de Bugen de Afrontamiento de la muerte con las modificaciones de Schmidt (2007).
- Escala de miedo a la muerte de Collett - Lester modificada por Bayés y Limonero (1999).

Así mismo se procedió a la realización de un programa formativo especifico sobre la muerte, el morir y los cuidados paliativos en el que se incluían las siguientes estrategias metodológicas para la adquisición de conocimientos y cambio de actitudes ante la muerte y el morir de los participantes del estudio.

- Clases magistrales sobre los principales conceptos de los procesos al fin de la vida y discusión sobre los mismos.

- Lectura y análisis de textos científicos y casos clínicos reales publicados, relacionados con el proceso de morir y la muerte.

- Visionado del documental "El fin de la vida".

Los datos obtenidos han sido analizados mediante el programa estadístico SPSS versión 20.0 para Windows. Se realizó el análisis descriptivo calculando frecuencias absolutas, relativas y porcentajes para las variables cualitativas (sexo, tanatología, ciclo), y medias y desviaciones típicas para las cuantitativas (puntuación en la escala de miedo ante la muerte).

Para el análisis de las relaciones entre las variables miedo ante la muerte y sexo, formación y semestre, se aplicó la prueba de Wilcoxon para ver la significación estadística de dicha relación.

El equipo de investigadores hizo mención expresa a que la participación era totalmente voluntaria, confidencial y anónima según la ley de protección de datos a la que se ciñen en los consentimientos informados.

Así mismo los investigadores se comprometieron a facilitar los resultados obtenidos a aquellas personas de la institución que lo solicitasen.

\section{RESULTADOS}

La población de referencia de nuestro estudio fue la totalidad de alumnos que conformaban la Licenciatura de Enfermería que cursaban el primer semestre del curso académico 2012-2013, menos los que participaron en la adaptación de cuestionarios ( 40 alumnos y 11 profesores).

La media de edad de los participantes ha sido de 20,18 años, con un rango de edad que oscila desde los dieciocho, hasta los veinticuatro años, por lo que consideramos que se trata de una población joven. Este grupo es coincidente con la mayoría de estudios que se realizan con universitarios (Benbunan et al., 2007; Campos, Rojas, Armero, González, Pérez y Hernández, 2002; Claxton, Crain y Claxton, 2007; Schmidt et al., 2012).

Tabla 1. Constitución de la muestra distribuida por sexo y ciclo que realizan

\begin{tabular}{|c|c|c|c|c|c|c|c|c|c|c|}
\hline \multirow[b]{2}{*}{$\begin{array}{l}\text { Sexo } \\
\mathrm{n}^{\circ} \text { de Suj. } \\
\% \text { Sujetos }\end{array}$} & \multicolumn{2}{|c|}{ Ciclo 1} & \multicolumn{2}{|c|}{ Ciclo 3} & \multicolumn{2}{|c|}{ Ciclo 5} & \multicolumn{2}{|c|}{ Ciclo 7} & Total & $\%$ \\
\hline & \begin{tabular}{l}
\multicolumn{1}{c}{$\boldsymbol{\sigma}^{\lambda}$} \\
29 \\
10,59
\end{tabular} & $\begin{array}{l}+9 \\
74 \\
27,01\end{array}$ & $\begin{array}{c}\delta^{\pi} \\
15 \\
5,47\end{array}$ & $\begin{array}{l}+9 \\
74 \\
27,01\end{array}$ & $\begin{array}{c}\delta^{\pi} \\
10^{\pi}, 65\end{array}$ & $\begin{array}{l}\text { 9 } \\
31 \\
11,31\end{array}$ & $\begin{array}{l}\delta^{\pi} \\
7 \\
2,56\end{array}$ & $\begin{array}{l}+9 \\
34 \\
12,41\end{array}$ & 274 & $100 \%$ \\
\hline
\end{tabular}

Fuente: Elaboración propia

La muestra se compuso principalmente de mujeres, siendo el $22,3 \%$ de los alumnos participantes hombres, frente al $77,7 \%$ de mujeres. Datos muy semejantes podemos encontrarnos en estudios que siguen la misma línea de investigación y que trabajan con universitarios, como el de Edo-Gual, Tomas-Sábado y Ardilla (2011). Este estudio también coincide en la distribución por ciclos de la licenciatura. 
Tabla 2. Muestra distribuida por el ciclo que realizan y si han cursado o no Tanatología según la población total de referencia y teniendo en cuenta solo al alumnado de $5^{\circ}$ y $7^{\circ}$ ciclo

\begin{tabular}{|c|c|c|c|c|c|c|c|c|c|c|}
\hline & \multicolumn{2}{|c|}{ Ciclo 1} & \multicolumn{2}{|c|}{ Ciclo 3} & \multicolumn{2}{|c|}{ Ciclo 5} & \multicolumn{2}{|c|}{ Ciclo 7} & Total & $\%$ \\
\hline $\begin{array}{l}\text { Tanatología } \\
\mathrm{n}^{\circ} \text { de Suj. } \\
\% \text { Sujetos } \\
\mathbf{N}^{\circ} \text { de sujeto } \\
\% \text { de sujetos }\end{array}$ & $\begin{array}{l}\text { SI } \\
- \\
\text { lo c } \\
\text { o d }\end{array}$ & $\begin{array}{l}\mathrm{NO} \\
103 \\
37,59 \\
7^{\circ} \text { ciclo } \\
7^{\circ} \text { ciclo }\end{array}$ & $\begin{array}{l}\text { SI } \\
-\end{array}$ & $\begin{array}{l}\mathrm{NO} \\
89 \\
32,48\end{array}$ & $\begin{array}{l}\text { SI } \\
3 \\
1,09 \\
3 \\
3,66\end{array}$ & $\begin{array}{l}\text { NO } \\
38 \\
13,87 \\
38 \\
46,34\end{array}$ & $\begin{array}{l}\text { SI } \\
24 \\
8,76 \\
24 \\
29,27\end{array}$ & $\begin{array}{l}\text { NO } \\
17 \\
6,20 \\
17 \\
20,73\end{array}$ & $\begin{array}{l}274 \\
82\end{array}$ & $\begin{array}{l}100 \% \\
100 \%\end{array}$ \\
\hline
\end{tabular}

Fuente: Elaboración propia

La variable religión sería importante el haberla contemplado, ya que esta podría influir en las respuestas de los sujetos. Tal y como hemos advertido al revisar la literatura existente, esta, en muchos casos, avala la relación entre las actitudes negativas ante la muerte, las creencias y la práctica religiosa (Edo-Gual, Tomas-Sábado y Ardilla, 2011; Tomas-Sábado y Gómez-Benito, 2003; Siracusa et al., 2011; Tomas-Sábado y Limonero, 2006). Al comprobar que existía un amplio abanico de religiones el equipo de investigadores considero no tenerla en cuenta.
La adaptación de escalas se utilizó buscando una equivalencia conceptual en los términos utilizados, valorando a su vez la pertinencia de los diversos ítems en ese contexto cultural.

Los resultados obtenidos en el análisis estadístico muestran para las diferentes escalas y grupos, una consistencia interna favorable con un alfa de Cronbach comprendido entre 0,796 y 0,916. Estos valores están considerados como buenos o incluso excelentes. Véase la tabla 3 que se presenta a continuación.

Tabla 3. Alfa de Cronbach observado en los las distintas escalas y grupos

\begin{tabular}{lcc}
\hline \multicolumn{1}{c}{ Escala } & Grupo experimental & Grupo Control \\
\hline $\begin{array}{l}\text { Escala de ansiedad ante la muerte de } \\
\text { Thorson y Powell }\end{array}$ & 0,843 & 0,796 \\
$\begin{array}{l}\text { Escala de afrontamiento ante la muerte } \\
\text { de Burgen }\end{array}$ & 0,854 & 0,869 \\
$\begin{array}{l}\text { Escala de miedo ante la muerte de } \\
\text { Collett y Lester }\end{array}$ & 0,905 & 0,916 \\
\hline
\end{tabular}

Fuente: Elaboración propia

\section{DISCUSIÓN}

Lo anteriormente expuesto nos indica que nuestros instrumentos, tienen un grado aceptable de consistencia interna para medir el miedo, la ansiedad y el afrontamiento, en nuestra población de estudio. A su vez, podemos decir que es muy semejante a la encontrada en múltiple literatura que utiliza estos instrumentos en sus investigaciones (Abdel-Khalek y Lester, 2004; Espinosa, Sanhueza y Barriga ,2011 y TomasSábado, Limonero y Abdel-Khalek, 2007) entre otros. De esta forma se puede mencionar que los instrumentos utilizados son validos y confiables en otros contextos de investigación, relacionados con el morir y su proceso (Neimeyer, 1997; Vargas y Cervantes, 2009).

De manera general, tras la implementación de nuestro programa específico acerca de la muerte y su proceso, los alumnos del grupo experimental han modificado el miedo, la ansiedad y el afrontamiento de manera positiva, por otra parte y tal y como se esperaba, aquellos integrantes pertenecientes al grupo control, no han modificado su parámetros iníciales en cuanto a las actitudes referidas anteriormente de este modo, y tras el tratamiento de datos, podemos mencionar que nuestro programa específico acerca de la muerte y su proceso, es efectivo para cambiar las actitudes que se presentan cuando la persona se tiene que enfrentar a la muerte, en nuestra población de estudio.

Cuando se procede a comparar los resultados obtenidos en relación al miedo que la persona presenta ante su propio morir o el de la persona más querida, o el proceso de morir personal o el ajeno mediante la escala de Collett Lester de miedo ante la muerte en las diferentes etapas del estudio, observamos que no encontramos diferencias significativas en ninguno de los grupos sociodemográficos estudiados: sexo, ciclo y formación o ausencia de la misma en tanatología.

Se observa el hecho de que las mujeres presentan mayor índice de miedo que los varones en las diferentes subescalas. La presencia de este fenómeno repetitivo, puede señalar según varios autores que nos pudiésemos encontrar ante un hecho universal (Pollak, 1979; Edo-Gual, TomasSábado y Ardilla Herrero, 2011).

Así mismo, los resultados del estudio muestran claramente la diferencia, entre muerte y proceso de morir, la muerte propia y la muerte de otro, respecto a los parámetros emocionales evaluados.

En cuanto al estudio de la competencia percibida ante la muerte, mediante el uso la escala de afrontamiento de Bugen (1981), cuando se procede a comparar los resultados obtenidos en las diferentes etapas del estudio, observamos la ausencia de diferencias significativas en relación al afrontamiento ante la muerte y a las diferentes variables sociodemográficas estudiadas.

Cabe hacer mención a que el plan de intervención ha sido eficaz en cuanto al aumento del afrontamiento ante el proceso de morir, medido con la escala de Bugen, puesto que los valores globales entre ambas fases del estudio, han variado positivamente.

Al proceder a evaluar la ansiedad a través de la escala de ansiedad ante la muerte de Thorson y Powell, en su versión modificada de la original escala (EAM) creada por Donald Templer en 1970 podemos decir que el programa de intervención ha sido efectivo.

Los resultados de nuestro estudio no muestran diferencia significativas entre sexos, lo que no es coincidente con resultados encontrados por el resto de autores (Colell, 2005; Schmidt et al., 2007; Tomás Sábado y Gómez Benito, 2003). Este fenómeno aparentemente anormal pudiera ser motivado, a nuestro parecer por el singular comportamiento que presenta esta población en referencia a su visión y convivencia en el día a día con la muerte. Hecho en la actualidad el equipo de investigadores sigue trabajando en él. 
En nuestro estudio, no encontramos diferencias significativas entre los alumnos que cursaban los diferentes ciclos de enfermería. Este hecho no es coincidente con investigaciones realizados por autores como Johansson y Lally (1991), Tomas Sábado y Guix Llistuella, (2001), los cuales coinciden en decir, que las experiencias clínicas que implican la muerte de alguno de los pacientes durante las prácticas clínicas que los alumnos realizan, habrían aumentado su capacidad de afrontamiento a la muerte.

De igual forma, no se observaron diferencias significativas entre el alumnado de enfermería que había tenido formación en tanatología con respecto a los que no habían cursado esa materia. Señalamos que la escasez de sujetos con formación en tanatología que participaron en el estudio, se debe a que dicha formación, solo se ofrece en su plan de estudios a alumnos de los ciclos cinco y siete, y es de carácter optativo. Por lo que nuestros resultados deben ser considerados con suma cautela.

Así pues, acabar mencionando que uno de los principales desafíos que afecta actualmente a los estudios de ciencias de la salud, es la necesidad de formación específica que sirva para desenvolverse ante problemas cotidianos en su ejercicio profesional. En este caso las universidades tienen la obligación de preparar a sus alumnos teniendo en cuenta aquello que se van a encontrar en la realidad y que en muchos casos, no tiene que ver con lo enseñado en la teoría. Por este motivo se hace evidente que el personal que atiende a enfermos terminales y trabaja día a día rodeado de pacientes sin expectativas de curación, necesita a través de una formación específica, canalizar sus miedos y emociones, relacionados con la muerte.

Se concluye que:

- La consistencia interna de las tres escalas tanto en el grupo control como el experimental es considerada como buena o excelente.

- El programa de formación ante la muerte y el morir ha sido efectivo en cuanto a la disminución del miedo y la ansiedad y el aumento de afrontamiento ante la muerte de los estudiantes participantes en el estudio.

- La inclusión de los contenidos del programa de intervención en el Plan de estudios de Enfermería de la UNACAR, podría expresarse, en un futuro laboral, como una mejora de los cuidados en enfermos terminales.

\section{REFERENCIAS BIBLIOGRÁFICAS}

Abdel-Khalek, A., y Lester, D. (2004). The factorial structure of the Arabic version of the Revised Collett-Lester Fear of Death Scale. Death studies,28(8), 787-793.

Benbunan-Bentata, B., Alfaya, M.M., Chocrom, S., Cruz, F., Villaverde, C., y Roa, J.M. (2007). El impacto emotivo del hospital, implicaciones en la formación universitaria de los estudiantes de enfermería. Rev ROL Enfermería, 28, 675682.

Bugen, L. A. (1980-1981). Coping: Effects of death education. Journal of Death and Dying,11(2), 175-183.

Campos Aranda, M., Rojas Alcántara, P., Armero Blanco, D., González Quijano, A., Pérez, G., y Hernández Rojas, V.M. (2002). Demandas ante un proceso de muerte. Enfermería Global, 1, 22-5.

Claxton-Oldfield. S., Crain. M., y Claxton-Oldfield, J. (2007).Death anxiety and death competency: the impact of a palliative care volunteer training program. Am J Hosp Palliat Care,23, 464-8.

Colell Brunet, R. (2005). Análisis de las actitudes ante la muerte y el enfermo al final de la vida en estudiantes de enfermería de Andalucía y Cataluña. (Tesis doctoral inédita). Universidad Autónoma, Barcelona.

Edo-Gual, M., Tomás-Sábado, J., y Aradilla-Herrero, A.(2011). Miedo a la muerte en estudiantes de enfermería. Enfermería Clínica, 21(3),129-135.

Espinosa Venegas, M., Sanhueza Alvarado, O., y Barriga, O. (2011). Validación de la Escala de Miedo a la Muerte de Collett-Lester en una muestra de estudiantes de
Enfermería. Rev. Latino-Am. Enfermagem, 19(5).(10 Pantallas)Disponible en: http://www.scielo. $\mathrm{br} / \mathrm{pdf} / \mathrm{rlae} / \mathrm{v} 19 \mathrm{n} 5 / \mathrm{es}$ 15.pdf

Gala León, F. J., Lupiani Giménez, M., Raja Hernández, R., Guillén Gestoso, C., González Infante, J. M., Villaverde Gutiérrez, M. C., y Alba Sánchez, I. (2002). Actitudes psicológicas ante la muerte y el duelo. Una revisión conceptual. Cuadernos de Medicina Forense, 30, 39-50.

Gálvez González, M. (2011). Cuando la familia lo vive, las palabras sobran. El modelo de cuidados compartidos en la fase terminal del paciente crítico. Paraninfo Digital. Monográficos de Investigación en Salud. 11.Disponible en: http://www.index-f.com/para/n11-12/192p.php

García Caro, M.P. (2008). Pensar en Paliativo. Estudio cualitativo de la experiencia de profesionales médicos y enfermeros en la asistencia al enfermo terminal en Granada y provincia. (Tesis doctoral inédita). Universidad de Granada.

Johansson, N., y Lally, T. (1994). Effectiveness of a deatheducation program in reducing death anxiety of nursing students. Omega, 22, 25-33

Lamberth, J. (1982). Psicología Social. Madrid: Pirámide

Mason SR, Ellershaw JE. (2010). Undergraduate training in palliative medicine: is more necessarily better? Palliat Med 24:306-309.

Neimeyer, R. A. (1997). Métodos de evaluación de la ansiedad ante la muerte. Barcelona: Paidós.

Pollak, J.M. (1979). Correlates of death anxiety: a review of empirical studies. Omega J Death Dying, 10, 97-121

Sanz-Ortiz, J. (2006). ¿Es posible gestionar el proceso de morir? Voluntades anticipadas. Med Clin 126 (16), 620623.

Siracusa, C.F.(2010). Educación para la muerte: estudio sobre la construcción del concepto de muerte en niños de entre 8 a 12 años de edad, en el ámbito escolar. Propuesta de un programa de Intervención. (Tesis doctoral inédita). Universidad de Granada, Granada.

Siracusa, C.F., Cruz-Quintana, F., Pérez-Marfil, M.N., GarcíaCaro, M.P., Schmidt-Rio-Valle, J., y Vera-Martínez, M. (2011). Actitudes y afrontamiento ante la muerte en padres de niños de primaria. Psicología Conductual, 19(3), 627-642.

Schmidt Rio-Valle, J. (2007). Validación de la versión española de la Escala de Bugen de afrontamiento a la muerte y del perfil revisado de actitudes hacia la muerte. Estudio comparativo y transcultural. Puesta en marcha de un programa de investigación. (Tesis doctoral inédita). Universidad de Granada.

Schmidt Rio-Valle, J., Montoya, R., Campos, C.P., García-Caro, M.P., Prados, D. y Cruz Quintana, F. (2012). Efectos de un programa de formación en cuidados paliativos sobre el afrontamiento de la muerte. Revista Medicina Paliativa, 19(3), 113-20.

Templer, D.I. (1970). The construction and validation of a Death Anxiety Scale. Journal of General Psychology, 82, 165177.

Tomás-Sábado, J., y Gómez Benito, J. (2003). Variables relacionadas con la ansiedad ante la muerte. Revista de Psicología General y Aplicada, 56(3), 257-79.

Tomás-Sábado, J., y Guix Llistuella, E. (2001). Ansiedad ante la muerte. Efectos de un curso de formación en enfermeras auxiliares de enfermería. Enfermería Clínica, 11(3). 104109.

Tomás-Sábado, J., y Limonero, J. T. (2006) Religiousness and death anxiety. En Ambrose SD, editor. Religion and Psychology: New Research (pp. 107-22). New York: Nova Science Publishers.

Tomás Sábado, J., Limonero García, J. T., y AbdelKhalek, A. M. (2007).Spanish adaptation of the Collet-Lester fear of death scale. Death Studies, 31(3), 249-260.

Vargas, J. E., Cervantes, M., y Everardo, J. (2009). Estrategias de afrontamiento del Sida en pacientes diagnosticados como seropositivosIl, Psicología y Salud, 19(2), 215-221. 